\title{
FECAL TRANSPLANTATION: DIGESTIVE AND EXTRADIGESTIVE CLINICAL APPLICATIONS
}

\author{
MIHAELA FILIP ${ }^{1}$, VALENTINA TZANEVA ${ }^{2}$, DAN L. DUMITRASCU ${ }^{1}$ \\ 12nd Deptartment Internal Medicine, Iuliu Hatieganu University of Medicine and \\ Pharmacy, Cluj-Napoca, Romania \\ ${ }^{2}$ Trakia University Stara Zagora, Bulgaria
}

\begin{abstract}
Background and aim. Fecal transplantation or fecal material transplantation (FMT) became a hot topic in gastroenterology in recent years. Therefore it is important to disseminate the up-to-date information on FMT. The aim of the paper is to review the knowledge on FMT and its clinical applications.

Methods. An extensive review of the literature was carried out. Titles from Pubmed were searched and analyzed. A narrative review has been written with emphasis on indications of FMT in different conditions.

Results. The guidelines recommend FMT in relapsing infection with Clostridium difficile. Several attempts to use FMT in other conditions have been analyzed. Attempts were recorded in other bowel disorders like IBD, IBS, chronic constipation and even colorectal cancer. The attempt to change the microbiota by FMT in diabetes and obesity represent challenges for the future.

Conclusions. Fecal transplantation represents an important therapeutic method, intensively investigated these years. Beside the indication for persistent and recurrent Clostridium difficile infection, several attempts were undertaken in other intestinal diseases and in metabolic conditions. The efficiency of these applications has to be demonstrated.
\end{abstract}

Keywords: fecal transplantation, IBS, IBD, intestinal diseases

\section{Intestinal microbiota}

The term human gastrointestinal microbiota refers to the microbial population present in the digestive tract. On average, the intestinal microbiota comprises of 100 billion microbes, i.e. 10 times more than the cells in the human body [1].

Overall, the healthy gut microbiota (GM) is predominantly constituted by the phyla Firmicutes and Bacteroidetes. They are followed by the phyla Actinobacteria and Verrucomicrobia.

Even though this general profile remains constant, intestinal microbiota can be different at different time intervals in the same anatomical place in the same person, due to environmental changes. From the esophagus to the rectum, there will be a marked difference in diversity and

Manuscript received: 05.11.2017

Accepted: 06.12.2017

Address for correspondence: ddumitrascu@umfcluj.ro number of bacteria ranging from $10^{1}$ per gram of contents in the esophagus and stomach, to $10^{12}$ per gram of contents in the colon and distal gut [2,3].

The gut microbiome is established during the first year of life and is influenced by multiple host and external factors including diet and antibiotics [4]. The intestinal microbiota of neonates is characterized by a low diversity and relative dominance of Proteobacteria and Actinobacteria. Then the microbiota becomes more diverse with the emergence of Firmicutes and Bacteroidetes domination, which characterizes the adult microbiota.

Microbiota produces metabolites that can have positive effects on the host, including anti-inflammatory and antioxidant activity, bowel barrier function regulation, and vitamin production and energy sources. Commensal bacteria inhabiting the human intestine participate in the development and maintenance of gut immunologic, sensory and motor functions. Under normal conditions, the 
gastrointestinal tract provides a stable habitat for commensal bacteria that supports its structural and functional integrity [5]. Enteric bacteria have many benefits for gut physiology, including protective, structural and metabolic implications.

The specific changes in the composition of gut microbiota have been associated not only with many gastrointestinal diseases but also with extra intestinal diseases: metabolic diseases, autoimmune diseases, allergic disorders, and neuropsychiatric disorders [6].

If in the case of infection with Clostridium difficile, inflammatory bowel disease or irritable bowel, the role of the gut microbiome has been established with certainty, its role in the extra digestive pathology is not yet fully elucidated, though the data accumulated so far are sufficient to confirm the involvement of the microbiome in a number of important extra intestinal diseases.

\section{Fecal transplantation or fecal material transplantation (FMT)}

The restoration of a normal intestinal microbiome can be achieved by the transfer of fecal material (FMT) from a healthy donor to the patient, in order to increase the intestinal microbial diversity.

The first known description of the use of feces as therapy was described by Ge Hong in 4th-century China for the treatment of any diseases, for example diarrhea $[7,8]$, but the method of transplantation was mentioned for the first time in 1958 by an Australian physician who administered patients fecal enemas from healthy people in order to repopulate the gut with beneficial bacteria Lactobacillus acidophilus and Bifidobacterium type bifidus which resulted in a rapid return to health [9]. Sporadically reports from patients trying to cure their bowel problems from feces of relatives may also be encountered.

Currently, the most common indication for fecal transplantation is the recurrent Clostridium difficile infection, but fecal transplantation is also being tested as a treatment for other gastrointestinal diseases, as well as certain non-gastrointestinal conditions including Parkinson's disease, fibromyalgia, chronic fatigue syndrome, multiple sclerosis, obesity, insulin resistance, metabolic syndrome, autism and more, though these have to be evaluated further in clinical trials $[6,10]$.

FMT is an easy technique that requires a healthy donor (at the beginning they were the patient's family members, nowadays - there are banks of donated feces for transplantation), previously investigated according to a well established protocol in order to eliminate the potential risk factors, such as the presence of some infection, viruses, parasites, hepatitis viruses, HIV or other bacterial pathogens [11].

A fecal suspension can be administered by nasogastric or nasoduodenal tube, colonoscope, enema or capsule [6].

Nasoduodenal route of administration is the most difficultly tolerated method by patients. Colonoscopy allows direct assessment of the colonic mucosa and evaluation of disease severity and exclusion of coexistent pathology. Enema administration is effective, cheap and safe and carries less procedural or institutional admission costs [11].

Oral administration is likely to make FMT even more favorable in terms of cost-benefit, but the use of capsules requires the patients' cooperation and it is limited for patients with dysphagia or gastrointestinal dysmotility [12].

Recent data have shown that oral administration of donor material encapsulated lyophilized resulted in rates of healing comparable to colonoscopy.

Prior to FMT, microbiome intestinal patient fecal samples showed declines in diversity and abundance of Firmicutes and Bacteroidetes, with concurrent increases in members of the Proteobacteria, specifically Enterobacteriaceae. After the transplantation, Firmicutes showed rapid increase in relative abundance and did not vary significantly over time. Conversely, Bacteroidetes only showed significant increases in abundance after one month post-FMT, corresponding to significant increases in the community attributable to the donors [13].

\section{Infection with Clostridium difficile}

Clostridium difficile (CD), although naturally found in the intestinal microbial ecosystem, can cause particularly dangerous infections from pre-exposure to broad-spectrum antibiotics, as they disrupt the balance of intestinal flora that protects colonization with Clostridium difficile. The evolution of the infection depends on the speed and correctness of the established treatment, but there is a risk of relapse of the disease even under the conditions of a correct and complete treatment [14].

Fecal microbiota transplantation (FMT) is a new, safe and effective treatment of recurrent CDI with reported success rates of up to $90 \%[15,16]$.

Numerous studies exist on the fecal microbiota analysis of patients with CDI recurrence and donors, which show that the CDI is associated with reduced changes of diversity and composition in the fecal microbiota.

The members of Streptococcaceae, Enterococcaceae or Enterobacteriaceae were increased and butyrateproducing (Lachnospiraceae and Ruminococcaceae) were reduced in the samples from RCDI patients before FMT compared to post FMT and healthy donor samples [17].

Recent research showed that approximately $85 \%$ of affected patients can be successfully treated by FMT compared to only about $30 \%$ treated conventionally with vancomycin [18]. Fecal microbiota transplantation (FMT) is a highly effective therapy for persistent and recurrent Clostridium difficile infection [19].

With regard to the way of administration, frozen preparations showed that their administration is as effective as other ways of administration. A meta-analysis among 
patients with recurrent or refractory CDI, have shown that frozen FMT is as effective as fresh FMT, making it an option for the decrease of the potential risks using the other ways of administration [20]. A single administration of encapsulated, freeze-dried fecal microbiota from a healthy donor was highly successful in treating antibioticrefractory R-CDI syndrome [21].

\section{Irritable bowel syndrome}

The irritable bowel syndrome (IBS) presents with association of chronic and recurrent symptoms such as constipation, diarrhea, bloating and/or abdominal pain, having no detectable biochemical or structural abnormalities [22]. The pathogenesis of irritable bowel syndrome is multifactorial and is not fully understood, it is believed to involve a complex interplay among the intestinal microbiota, immune system, and brain-gut axis $[23,24]$.

The gut flora can produce a range of neuroactive molecules, such as acetylcholine, catecholamines, $\gamma$-aminobutyric acid, histamine, melatonin and serotonin which are essential for regulating peristalsis and sensation in the gut [25]. Recognition of the relationship between the microbial-gut-brain axis and the neuroimmune systems provides a new approach for better understanding and management of these disorders [25].

A new strategy for the treatment of this multifactorial disease is represented by manipulation of gut microbiota. Because FMT is an inexpensive and easy treatment, it might be an option for treating IBS, but standardized controlled trials are necessary to ascertain which patients are eligible, and also the method of administration of the donor's microbiota [11].

A study which included patients, diagnosed with IBS based on Rome III Diagnostic Criteria, who received fecal materials via colonoscopy, showed that the FMT for patients with IBS is safe, and relatively effective method, which improved the psychological status of IBS patients [26].

Many attempts have been made to modulate the gut bacterial composition, presuming that the increase of bacterial species considered as beneficial (Lactobacilli and Bifidobacteria) associated with the decrease of those considered harmful (Clostridium, Escherichia coli, Salmonella, Shigella and Pseudomonas) should attenuate IBS symptoms. Fecal transplant is a therapeutic option for those patients with a disturbed gut ecosystem, but data on IBS are few and randomized, placebo-controlled studies are required [11].

\section{Ulcerative colitis and Crohn disease}

Since the 1900s, physicians reported that bacteria may play a role in colitis [27]. Ulcerative colitis and Crohn's disease are chronic inflammatory diseases of the intestines and the etiology of inflammatory bowel diseases involves an activation of the mucosal immune system as a result of dysbiosis, resulting in chronic inflammation and the development of mucosal lesions [28]. It was proved that the fecal bacterial flora of IBD patients is different from the one of the healthy individuals [29]. In areas of active inflammation a decreased bacterial load is present [8]. Nowadays, the treatment focuses on the modulation of the immune system and is limited by side effects and a lack of efficacy [30].

The effect of FMT has mainly been investigated in ulcerative colitis patients. Two randomized controlled trials involving 75 patients with active UC and 50 patients with mild to moderately active ulcerative colitis had mixed results. In the first study, weekly FMT or water enema were administered for 6 weeks, with remission occurring in $24 \%$ of the patients treated with FMT, compared with $5 \%$ in the second group treated with water enema. In the second study, FMT was administered via nasoduodenal tube, administered initially and after 3 weeks. There was no difference in clinical and endoscopic remission between the two groups in 37 patients who could be traced. These different results may be due to differences in routes of administration, dosing schedules, stool donors or concomitant therapies [8].

In ulcerative colitis, the underlying pathophysiology may favor distal as opposed to proximal FMT administration [30].

Future trends are the use of defined stable microbiota preparations, in particular oral preparations, which will enable better and larger controlled trails for investigating FMT in inflammatory bowel diseases [31].

\section{Colorectal cancer}

The gut microbiota constitutes a natural defensive barrier to infection and plays a large role in maintaining gut homeostasis [32].

Many changes in the gut microbiota composition have been reported in colorectal cancer. The pro-carcinogenic properties of bacteria (genotoxicity, inflammation and oxidative stress) and dysbiosis are now better understood and are considered to be possible links between bacterial microbiota and colorectal carcinogenesis. Some bacterial species have been identified and suspected to play a role in colorectal carcinogenesis, such as Bacteroides fragilis, Streptococcus bovis, Clostridium septicum, Helicobacter pylori, Enterococcus faecalis, Escherichia coli and Fusobacterium spp. [33].

Colorectal cancer (CRC) is a complex association of tumoral cells, non-neoplastic cells (stromal cells) and a large number of micro-organisms. In addition to known risk factors (fat-rich diets, obesity, population, living in a developed country) and the uncontrolled cellular proliferation, in recent decades a growing attention has been given to the role of microbial infection in carcinogenesis, and microbes are suspected to be involved in approximately 
$20 \%$ of cancers, especially CRC [34].

During their phylogenetic evolution, bacteria progressively acquired virulence factors that conferred pathogenicity. Most of the disease-promoting and procarcinogenic effects of pathogens depend on these virulence factors [35].

Regarding the involvement of bacterial virulence factors (genotoxin production), it is increasingly mentioned that microbial derived microbial metabolites strongly influence the development of CRC [36]. A number of studies have revealed a link between Fusobacterium and CRC [37,38], observation supported by the 16S DNA sequenced analysis of colorectal microbe, which showed high proportion of Fusobacterium genus: Fusobacterium nucleatum, Fusobacterium mortiferum and Fusobacterium necrophorum in tumor tissue, changes accompanied by a significant reduction of Firmicutes and Bacteroidetes [39].

The use of fecal transplantation protocols could replace CRC-associated dysbiosis and thus restore eubiosis in chronic diseases, helping to reduce activation of inflammatory, proliferative and pro-carcinogenic pathways and microbiota-induced genotoxicity. Even if fecal transplantation has not been well studied in CRC, future transplantation studies represent an important next step in this line of research [35].

\section{Obesity and metabolic syndrome}

Obesity is an imbalance between intake energy (intake value of food ingested) and energy consumption, characterized by excessive adipose tissue deposition. Metabolic syndrome is characterized by a constellation of signs such as central obesity, hypertension, dyslipidemia and hyperglycemia that increases one's risk for developing diabetes mellitus and heart disease [40].

In addition to food intake, that has an essential contribution to the physiology of obesity, intestinal microbiota is the second major factor behind obesity, modulating nutrient absorption and energy regulation [41].

Obesity is a major risk factor for diabetes, hypertension, and metabolic syndrome. Gut microbiota makes a great contribution to the pathogenesis of obesity and related diseases [42]. Gut microbiota is involved in energy harvest through regulating the diverse aspects of cellular differentiation and gene expression, synthesizing micronutrients, breaking down complex dietary macromolecules, fermenting indigestible food substances and absorption of electrolytes [4].

Many studies showed that gut microbiota is different for obese and lean people, specifically in the proportion of Bacteriodetes and Firmicutes, mainly due to a reduced Bacteriodetes proportion in obese subjects $[43,44]$.

At present bariatric surgery is the only available treatment for morbid obesity that sustains substantial weight loss [45], but fecal microbiome transplantation (FMT) has been suggested as a new method of altering the gut microbiota that may lead to beneficial metabolic changes, though the evidence for FMT effectiveness in the treatment of obesity is preliminary $[46,47]$.

There are not many studies evaluating efficacy of FMT for the treatment of obesity. In a small doubleblind, randomized, controlled study, FMT from healthy lean donors to obese patients (with metabolic syndrome), showed an increase in insulin sensitivity, after fecal transplantation, which has been associated with increasing intestinal microbial flora, especially butyrate-producing bacteria - Roseburia intestinalis. It is possible that an increase in butyrate production may be responsible for the observed therapeutic effect of FMT [4]. Many studies show that nutrient availability modulates the composition and activity of the gut microbiota [48].

\section{Diabetes Mellitus}

Diabetes Mellitus (DM) is a metabolic disorder characterized by chronic hyperglycemia accompanied by alterations to carbohydrate, lipid and protein metabolisms, representing a global epidemic. Patients with diabetes have altered function of polymorphonuclear leukocytes, impaired bactericidal activity, alteration of the function of $\mathrm{T}$ lymphocytes and altered response to antigen exposure. Diabetes mellitus type 1 and type 2 present numerous possible long-term complications, generally proportional to the degree and duration of hyperglycemia [49].

As discussed above, the gut microbiota has an important role in normal intestinal function, implicitly in maintaining the host's health. It is well known that the GM lives in the intestine, therefore DM-induced intestinal and colon changes are likely to alter GM composition and gut microbiota modifications can affect intestinal motility and structure [50].

Type 2 diabetes has been linked to gut microbiota that differs from that found in a healthy individual, patients have a reduced level of butyrate-producing bacteria and more pathogenic bacteria. Patients show more Betaproteobacteria and reduced Firmicutes and Clostridia levels compared to healthy subjects [46].

A correlation between Bacteroidetes and Firmicutes ratio and plasma glucose concentration in patients with type 2 diabetes and obese patients has been observed, concluding that manipulation of microbial composition may present a new approach for preventing and treating obesity and type 2 diabetes. Fecal transplantation is currently being also considered a treatment for type 2 diabetes. In a study by Virenze et al., for evaluating the therapeutic benefit of fecal transplantation in obesity and diabetes, after 6 weeks of fecal transplantation, a $75 \%$ increase in insulin sensitivity was noted. However, additional studies are needed to monitor the benefits of using fecal transplantation as a therapeutic method in diabetes mellitus [51]. 


\section{Neuropsychiatric diseases}

Microbial formation occurs in early childhood in the first years of birth and reflects a combination of genetic factors, maternal health, delivery method, subsequent nutrition, maternal and postnatal exposure to antibiotics [52].

It is considered that the alteration of the intestinal microbe in the first few months of life may be the origin of serious neuropsychiatric illnesses that occur in adulthood such as: schizophrenia, autism, behavioral or cognitive disorders, depression, anxiety, Alzheimer's disease, multiple sclerosis, chronic fatigue syndrome [53].

Recent evidence suggests a potential role of the microbiota - gut-brain axis in neuropsychiatric disorders; there is a growing emphasis on the influence of intestinal bacteria on human health [54]. In the last few years, a lot of research has been carried out in this direction. The routes of this relation are not fully elucidated but include metabolic, humoral, immune, and neural pathways [55]. It was proved that the presence of an endotoxin, namely lipopolysaccharide, would have an influence in the modulation of the central nervous system. Moreover it is responsible for the production of inflammatory cytokines that modify the physiological brain activity [56].

Up to now, available evidence indicates that damage of gut microbiota plays an essential role in the development of a neuropsychiatric illness, but the mechanism through which it happens is not fully understood. A deeper assessment of the role of intestinal flora in the genesis and development of these diseases is necessary, which would lead to a new therapeutic approach [56].

Recently, the first case of use in fecal transplantation to obtain remission of digestive symptoms was reported in a girl diagnosed with chronic disease and epilepsy (under sodium valproate treatment until transplantation). After 20 weeks, efficacy was observed in preventing seizures in the absence of anti-epileptic drugs. Other studies on the use of fecal transplantation in patients with Parkinson's disease, multiple sclerosis, chronic fatigue syndrome, autism revealed improvement in gastrointestinal symptomatology after fecal transplant [57], a finding that could open a new window in the mechanism of the disease by focusing on the axis of the microbial gut - brain and inspiring a new treatment by remodeling the intestinal microbiota [58].

\section{Allergic diseases}

An interesting chapter is the relationship of intestinal microbe with allergic diseases. In recent decades, there has been an increase in the prevalence of allergic diseases, such as asthma, eczema and food allergies. Recent evidence has suggested that intestinal microbial changes play an important role in influencing immunological events that could lead to the development of allergic diseases. These can be modulated by various environmental factors, such as diet, antibiotics, and early-life microbial exposures [59].

Recent research shows that there are differences between the intestinal microbial flora of asthmatic children versus healthy children. Decreased relative abundance of Lachnospira, Veillonella, Faecalibacterium, and Rothia in early infancy was reported to be associated with increased asthma risk. Also, an association was found between changes in abundance of Faecalibacterium prausnitzii and decreasing of butyrate and propionate in the determination of eczema [59].

Changes in intestinal microbiota play an important role in influencing immunological events that could lead to the development of allergic diseases. The ability of the microflora to influence the immune response resulted in new approaches in therapy that uses these differences in microbiota for the therapy and prophylaxis in patients with allergy [60]. The use of FMT seems promising in restoring immune homeostasis by transferring a complex community of bacteria which is more stable and harbors a greater ability to colonize [61].

\section{Conclusions}

The gut microbiota is important in the pathogenesis of digestive or extra digestive diseases and the studies that investigate this link are of great interest at the present time.

There is a tremendous attention in fecal transplant research for a multitude of digestive and extra digestive disorders associated with intestinal dysbiosis such as cardiovascular diseases, non-alcoholic liver steatosis, neuropsychiatric diseases, chronic acne, autoimmune diseases, renal and biliary lithiasis, metabolic syndrome with insulin resistance and many other diseases. Until now, FMT is an effective therapeutic procedure for recurrent CDI. However, it is expected that, in addition to conventional approaches, FMT standardization will be set in the coming years and that its guidelines will expand.

\section{References}

1. Buford TW. (Dis)Trust your gut: the gut microbiom in agerelated inflammation, health, and disease. Microbiome. $2017 \mathrm{Jul}$ 14;5(1):80. doi: 10.1186/s40168-017-0296-0.

2. Jandhyala SM, Talukdar R, Subramanyam C, Vuyyuru H, Sasikala M, Nageshwar Reddy D. Role of the normal gut microbiota. World J Gastroenterol. 2015;21(29):8787-8803.

3. O'Hara AM, Shanahan F. The gut flora as a forgotten organ. EMBO Rep. 2006;7(7):688-693.

4. Tehrani AB, Nezami BG, Gewirtz A, Srinivasan S. Obesity and its associated disease: a role for microbiota? Neurogastroenterol Motil. 2012;24(4):305-311.

5. Di Mauro A, Neu J, Riezzo G, Raimondi F, Martinelli D, Francavilla R, et al. Gastrointestinal function development and microbiota. Ital J Pediatr. 2013 Feb 24; 39:15. doi: 10.1186/18247288-39-15.

6. Choi HH, Cho YS. Fecal Microbiota Transplantation: Current Applications, Effectiveness, and Future Perspectives. Clin Endosc. 2016;49(3):257-265.

7. Zhang F, Luo W, Shi Y, Fan Z, Ji G. Should we standardize the 1,700-year-old fecal microbiota transplantation? Am J Gastroenterol. 2012;107(11):1755-1756. 
8. Gupta S, Allen-Vercoe E, Petrof EO. Fecal microbiota transplantation:in perspective. Therap Adv Gastroenterol. 2016;9(2):229-239.

9. Eiseman B, Silen W, Bascom GS, Kauvar AJ. Fecal enema as an adjunct in the treatment of pseudomembranous enterocolitis. Surgery. 1958;44(5):854-859.

10. Brandt LJ. Fecal transplantation for the Treatment of Clostridium difficile Infection. Gastroenterol Hepatol (N Y). 2012;8(3):191-194.

11. Distrutti E, Monaldi L, Ricci P, Fiorucci S. Gut microbiota role in irritable bowel syndrome: New therapeutic strategies. World J Gastroenterol. 2016;22(7): 2219-2241.

12. Youngster I, Mahabamunuge J, Systrom HK, Sauk J, Khalili $\mathrm{H}$, Levin J, et al. Oral, frozen fecal microbiota transplant (FMT) capsules for recurrent Clostridium difficile infection. BMC Med. 2016 Sep 9;14(1):134. doi: 10.1186/s12916-016-0680-9.

13. Staley C, Vaughn BP, Graiziger CT, Singroy S, Hamilton MJ, Yao D, et al. Community dynamics drive punctuated engraftment of the fecal microbiome following transplantation using freeze-dried, encapsulated fecal microbiota. Gut Microbes. 2017;8(3):276-288.

14. Jenior ML, Leslie JL, Young VB, Schloss PD. Clostridium difficile Colonizes Alternative Nutrient Niches during Infection across Distinct Murine Gut Microbiomes. mSystems. $2017 \mathrm{Jul}$ 25;2(4). doi: 10.1128/mSystems.00063-17.

15. Kassam Z, Lee CH, Yuan Y, Hunt RH. Fecal microbiota transplantation for Clostridium difficile infection: systematic review and meta-analysis. Am J Gastroenterol. 2013;108:500 508.

16. Juszczuk K, Grudlewska K, Mikucka A, Gospodarek E. Fecal microbiota transplantation - methods of treatment of recurrent Clostridium difficile infections and other diseases. Postepy Hig Med Dosw (Online). 2017;71(0):220-226.

17. Song Y, Garg S, Girotra M, Maddox C, von Rosenvinge EC, Dutta A, et al. Microbiota dynamics in patients treated with fecal microbiota transplantation for recurrent Clostridium difficile infection. PLoS One. 2013 Nov 26;8(11):e81330. doi: 10.1371/ journal.pone.0081330.

18. Lübbert C, Salzberger B, Mössner J. Fecal microbiota transplantation. Internist (Berl). 2017;58(5):456-468.

19. Bang BW, Park JS, Kim HK, Shin YW, Kwon KS, Kwon HY, et al. Fecal Microbiota Transplantation for Refractory and Recurrent Clostridium difficile Infection: A Case Series of Nine Patients. Korean J Gastroenterol. 2017;69(4):226-231.

20. Tang G, Yin W, Liu W. Is frozen fecal microbiota transplantation as effective as fresh fecal microbiota transplantation in patients with recurrent or refractory Clostridium difficile infection: A meta-analysis? Diagn Microbiol Infect Dis. 2017;88(4):322-329. 21. Staley C, Hamilton MJ, Vaughn BP, Graiziger CT, Newman KM, Kabage AJ, et al. Successful Resolution of Recurrent Clostridium difficile Infection using Freeze-Dried, Encapsulated Fecal Microbiota; Pragmatic Cohort Study. Am J Gastroenterol. 2017;112(6):940-947.

22. Iacob T, Țățulescu DF, Dumitraşcu DL. Therapy of the postinfectious irritable bowel syndrome: an update. Clujul Med. 2017;90(2):133-138.

23. Aroniadis OC, Brandt LJ. Intestinal microbiota and the efficacy of fecal microbiota transplantation in gastrointestinal disease. Gastroenterol Hepatol (N Y). 2014;10(4):230-237.

24. Lacy BE, Chey WD, Lembo AJ. New and Emerging Treatment Options for Irritable Bowel Syndrome. Gastroenterol Hepatol (N
Y). 2015;11(4 Suppl 2):1-19.

25. Petra AI, Panagiotidou S, Hatziagelaki E, Stewart JM, Conti P, Theoharides TC. Gut-Microbiota-Brain Axis and Its Effect on Neuropsychiatric Disorders with Suspected Immune Dysregulation. Clin Ther. 2015;37(5):984-995.

26. Mizuno S, Masaoka T, Naganuma M, Kishimoto T, Kitazawa M, Kurokawa S, et al. Bifidobacterium-Rich Fecal Donor May Be a Positive Predictor for Successful Fecal Microbiota Transplantation in Patients with Irritable Bowel Syndrome. Digestion. 2017;96(1):29-38.

27. Kelly CR, Kahn S, Kashyap P, Laine L, Rubin D, Atreja A, et al. Update on Fecal Microbiota Transplantation 2015: Indications, Methodologies, Mechanisms, and Outlook. Gastroenterology. 2015;149(1):223-237.

28. Bak SH, Choi HH, Lee J, Kim MH, Lee YH, Kim JS, et al. Fecal microbiota transplantation for refractory Crohn's disease. Intest Res. 2017;15(2):244-248.

29. Sartor RB. Microbial influences in inflammatory bowel diseases. Gastroenterology. 2008;134(2):577-594.

30. Lopez J, Grinspan A. Fecal Microbiota Transplantation for Inflammatory Bowel Disease. Gastroenterol Hepatol (N Y). 2016;126):374-379.

31. Kump P, Högenauer C. Any Future for Fecal Microbiota Transplantation as Treatment Strategy for Inflammatory Bowel Diseases? Dig Dis. 2016;34 Suppl 1:74-81.

32. Boleij A, Tjalsma H. Gut bacteria in health and disease: a survey on the interface between intestinal microbiology and colorectal cancer. Biol Rev Camb Philos Soc. 2012;87:701-730.

33. Gagnière J, Raisch J, Veziant J, Barnich N, Bonnet R, Buc E, et al. Gut microbiota imbalance and colorectal cancer. World J Gastroenterol. 2016;22(2):501-518.

34. Zur Hausen H. The search for infectious causes of human cancers: where and why. Virology. 2009;392:1-10.

35. Schwabe RF, Jobin C. The microbiome and cancer. Nat Rev Cancer. 2013;13:800-812.

36. Louis P, Hold GL, Flint HJ. The gut microbiota, bacterial metabolites and colorectal cancer. Nat Rev Microbiol. 2014;12:661-672.

37. Castellarin M, Warren RL, Freeman JD, Dreolini L, Krzywinski M, Strauss J, et al. Fusobacterium nucleatum infection is prevalent in human colorectal carcinoma. Genome Res. 2012;22(2):299-306.

38. Keku TO, McCoy AN, Azcarate-Peril AM. Fusobacterium spp. and colorectal cancer: cause or consequence? Trends Microbiol. 2013;21(10):506-508.

39. Kostic AD, Gevers D, Pedamallu CS, Michaud M, Duke F, Earl AM, et al. Genomic analysis identifies association of Fusobacterium with colorectal carcinom. Genome Res. 2012;22(2):292-298.

40. Hoffman EL, VonWald T, Hansen K. The metabolic syndrome. S D Med. 2015;Spec No: 24-28.

41. Carlucci C, Petrof EO, Allen-Vercoe E. Fecal Microbiotabased Therapeutics for Recurrent Clostridium difficile Infection, Ulcerative Colitis and Obesity. EBioMedicine. 2016;13:37-45.

42. Drapkina OM, Korneeva ON. Gut microbiota and obesity: Pathogenetic relationships and ways to normalize the intestinal microflora. Ter Arkh. 2016;88(9):135-142.

43. Armougom F, Henry M, Vialettes B, Raccah D, Raoult D. Monitoring bacterial community of human gut microbiota reveals an increase in Lactobacillus in obese patients and Methanogens in anorexic patient. PLoS One. 2009 Sep 23;4(9):e7125. 
doi: 10.1371/journal.pone.0007125.

44. Samuel BS, Gordon JI. A humanized gnotobiotic mouse model of host-archaeal-bacterial mutualism. Proc Natl Acad Sci U S A. 2006;103:10011-10016.

45. Buchwald H, Avidor Y, Braunwald E, Jensen MD, Pories W, Fahrbach K, et al. Briatric surgery: a systematic review and metaanalysis. JAMA. 2004;292(14):1724-1737.

46. Jayasinghe TN, Chiavaroli V, Holland DJ, Cutfield WS, O'Sullivan JM. The New Era of Treatment for Obesity and Metabolic Disorders: Evidence and Expectations for Gut Microbiome Transplantation. Front Cell Infect Microbiol. 2016 Feb 19;6:15. doi: 10.3389/fcimb.2016.00015.

47. Smits LP, Bouter KE, de Vos WM, Borody TJ, Nieuwdorp M. Therapeutic potential of fecal microbiota transplantation. Gastroenterology. 2013;145(5):946-953.

48. Duranti S, Ferrario C, van Sinderen D, Ventura M, Turroni F. Obesity and microbiota: an example of an intricate relationship. Genes Nutr. 2017 Jun 15;12:18. doi: 10.1186/s12263-017-0566-2. 49. Mauri-Obradors E, Estrugo-Devesa E, Jané-Salas E, Viñas M, López-López J. Oral manifestations of Diabetes Mellitus. A systematic review. Med Oral Patol Oral Cir Bucal. 2017;22(5):e586-e594.

50. Zhao M, Liao D, Zhao J. Diabetes-induced mechanophysiological changes in the small intestine and colon. World J Diabetes. 2017;8(6):249-269.

51. Larsen N, Vogensen FK, van den Berg FW, Nielsen DS, Andreasen AS, Pedersen BK, et al. Gut microbiota in human adults with type 2 diabetes differs from non-diabetic adults. PLoS One. 2010 Feb 5;5(2):e9085. doi: 10.1371/journal.pone.0009085. 52. Azad MB, Konya T, Maughan H, Guttman DS, Field CJ, Chari RS, et al. Gut microbiota of healthy Canadian infants: profiles by mode of delivery and infant diet at 4 months. CMAJ. 2013;185(5):385-394.

53. Dash SR. The microbiome and brain health: what's the connection? Medscape. 2015 Mar 24. Available from: https:// www.medscape.com/viewarticle/841748

54. Rieder R, Wisniewski PJ, Alderman BL, Campbell SC. Microbes and mental health: A review. Brain Behav Immun. 2017;66:9-17.

55. Burokas A, Moloney RD, Dinan TG, Cryan JF. Microbiota regulation of the Mammalian gut-brain axis. Adv Appl Microbiol. 2015;91:1-62.

56. Mangiola F, Ianiro G, Franceschi F, Fagiuoli S, Gasbarrini G, Gasbarrini A. Gut microbiota in autism and mood disorders. World J Gastroenterol. 2016;22(1):361-368.

57. Xu MQ, Cao HL, Wang WQ, Wang S, Cao XC, Yan F, et al. Fecal microbiota transplantation broadening its application beyond intestinal disorders. World J Gastroenterol. 2015;21(1):102-111.

58. He Z, Cui BT, Zhang T, Li P, Long CY, Ji GZ, et al. Fecal microbiota transplantation cured epilepsy in a case with Crohn's disease: The first report. World J Gastroenterol. 2017;23(19):35653568 .

59. Chung KF. Airway microbial dysbiosis in asthmatic patients: A target for prevention and treatment? J Allergy Clin Immunol. 2017;139(4):1071-1081.

60. Maksimova OV, Gervazieva VB, Zverev VV. Intestine microbiota and allergic diseases. $\mathrm{Zh}$ Mikrobiol Epidemiol Immunobiol. 2014;(3):49-60.

61. Reynolds LA, Finlay BB. A case for antibiotic perturbation of the microbiota leading to allergy development. Expert Rev Clin Immunol. 2013;9(11):1019-1030. 